\title{
La literatura en la ontología social de John Searle
}

\section{Literature within John Searle's Social Ontology}

\author{
Rodrigo Guijarro Lasheras \\ Universidad Complutense de Madrid
}

\section{RESUMEN}

Estas páginas se proponen mostrar que el paradigma de la teoría de la construcción de la realidad social propuesto por John Searle en las últimas décadas permite derivar importantes elementos a la reflexión sobre literatura. La distinción entre «hechos sociales» y «hechos institucionales» supone ciertamente un sólido cimiento sobre el que articular una taxonomía de algunas de las principales aproximaciones a la ontología de la literatura. Un trabajo en esta línea de Vitor Guerreiro (2012) sobre filosofía de la música sirve de inspiración a la reflexión que se presenta aquí sobre el campo literario.

Palabras Clave: Ontología de la literatura; Searle; poética; hechos sociales e institucionales.

\begin{abstract}
John Searle has established one of the most appealing frameworks of the last decades regarding social ontology. The aim of this article is to initiate its application on the field of literary studies and ontology of literature. In that order, the distinction between social and institutional facts will serve as the backbone from which we could raise a taxonomy of different critical approaches towards the classic question of what literature is. For that purpose, we will also take account of recent ideas developed in philosophy of music studies, among which the article by Vitor Guerreiro (2012) will be an important source of inspiration for our reflection about the ontology of literature.
\end{abstract}

Key words: Ontology of Literature; Searle; Poetics; Social and Institutional Facts.

\section{INTRODUCCIÓN}

Que un término o un concepto se emplee de muy diversas maneras no quiere decir que se pueda emplear de cualquier modo. Con esta paráfrasis del crítico británico Terry Eagleton (2013: 48) podemos dar pie a la propuesta que se desarrollará a lo largo de estas páginas. El objetivo de este trabajo es así el de arrojar una nueva $-\mathrm{y}$, esperemos, esclarecedora- perspectiva sobre la

Copyright: (C) 2016 CSIC. Este es un artículo de acceso abierto distribuido bajo los términos de una licencia de uso y distribución Creative Commons Attribution (CC-by) España 3.0. 
recurrente cuestión de la ontología de la literatura a través del sustento teórico que nos proporciona la última producción de John Searle. Un artículo de Vitor Guerreiro (2012) novedoso en este sentido, si bien centrado en el concepto de música, nos servirá de apoyo a lo largo de estas páginas. Tras resumir los aspectos de la teoría searleana que consideramos significativos para el estudio de la literatura (secciones 1, 2 y 3), comentaremos brevemente la derivación que ha tenido este andamiaje teórico en la filosofía de la música (4 y 5) para concluir con una propuesta sobre la ontología de la literatura basada en los conceptos de hechos sociales e institucionales $(6,7$ y 8$)$.

\section{UN BREVE RECORRIDO POR EL PENSAMIENTO SEARLIANO}

La producción filosófica de John R. Searle muestra interés por un vasto repertorio temático. No obstante, podemos distinguir como mínimo tres periodos en su desarrollo filosófico. Tras el hito inaugural que supuso su teoría de los actos de habla, Searle dirige sus investigaciones hacia la filosofía de la mente. Es este «segundo Searle» el que, entre otras muchas cosas, desarrolla el concepto de intencionalidad. Finalmente, producto de una cohesionada evolución, se sitúa la tercera etapa de su producción, centrada en la ontología social. Abarca esta un periodo productivo que cubre desde 1995 a la actualidad. Será el pensamiento desarrollado en este lapso temporal el que emplearemos como marco básico en nuestro análisis. Particularmente, dos libros resultan imprescindibles para comprender la propuesta que aquí se planteará: $L a$ construcción de la realidad social (1997) [1995 edición inglesa], y Making the Social World. The Structure of Human Civilization (2011). No nos detendremos en la copiosa bibliografía crítica y exegética que estos textos han generado, si bien tres estudios monográficos nos resultarán muy relevantes para explicar las nociones capitales contenidas en estos libros, a saber: Nick Fotion (2000) y Joshua Rust (2006 y 2009).

\section{LOS CONCEPTOS CLAVE I: HECHOS BRUTOS, SOCIALES E INSTITUCIONALES}

La cuestión central a la que Searle trata de responder es cómo construimos la realidad social objetiva en que vivimos; esto es, cómo pasamos de un mundo de moléculas, átomos, agua, montañas, metales a un mundo con presidentes, mesas, destornilladores, dinero y matrimonios - y literatura también-. Es innegable que «hay cosas que existen solo porque creemos que existen» (Searle, 1997: 21). De este modo, «¿cómo puede ser un hecho completamente objetivo que los trocitos de papel que hay en mi bolsillo sean dinero si algo es dinero solo porque creemos que es dinero?» (Searle, 1997: 22).

Podemos introducir así la primera distinción fundamental. La realidad, tal 
y como la experimentamos, está constituida por tres tipos de hechos. En primer lugar tenemos los hechos brutos. Los hechos brutos existen con independencia de los agentes y de cómo estos los conceptualicen o los piensen. No guardan por tanto ninguna dependencia respecto de nuestros estados mentales o creencias. El agua es $\mathrm{H}_{2} \mathrm{O}$ con independencia de lo que nosotros pensemos sobre ella. Y, si pensamos que es $\mathrm{H}_{3} \mathrm{O}$ o maná de los dioses, sencillamente nos equivocamos. El agua, las montañas, los pájaros, el granito, los árboles son ejemplos de hechos brutos.

Proseguiremos definiendo los hechos institucionales, que son un tipo particular de hecho social. Estos existen y son lo que son única y exclusivamente en virtud de que los pensemos como tales, con independencia de sus propiedades materiales. El caso paradigmático que Searle desarrolla con especial brillantez y profusión es el del dinero. Cualquier cosa puede ser dinero si es aceptada socialmente como tal. Ahora bien, es importante resaltar que el hecho institucional no requiere consciencia de ser creado para existir. Son muy lúcidas las reflexiones que realiza Searle (1997: 23) en torno a la invisibilidad e ingravidez de la realidad institucional, que es percibida por los agentes de forma tan natural como si de agua, montañas o nubes se tratara, esto es, como si los hechos institucionales fueran de la misma índole que los hechos brutos. Yendo un paso más allá, puede haber dinero en una sociedad que no tenga expresamente el concepto de dinero, o puede haber lenguaje en un grupo humano primitivo que no tuviera consciencia de su empleo.

Por su parte, los hechos sociales son aquellos que satisfacen dos condiciones: como los hechos institucionales, deben ser pensados como $\mathrm{X}$ para que puedan ser X, pero requieren también satisfacer determinadas propiedades en virtud de sus rasgos materiales. Searle escoge como caso paradigmático el sacacorchos. Para que un objeto pueda ser un sacacorchos tiene que, en primer lugar, reunir las características materiales necesarias que lo capaciten para la función propia del sacacorchos. Pero, evidentemente, no todo lo que puede sacar un corcho es un sacacorchos, de tal manera que el objeto en cuestión deberá satisfacer un segundo requisito, a saber, el ser concebido y pensado como objeto para sacar corchos por los agentes. Lo mismo podemos decir de cualquier utensilio.

Todo hecho bruto es ontológicamente objetivo, esto es, son siempre aquellos hechos cuyo «modo de existencia es independiente de cualesquiera perceptores o de cualquier estado mental» (Searle, 1997: 27). Por su parte, los hechos sociales y los hechos institucionales son siempre ontológicamente subjetivos. Es más, no tienen existencia más allá o con independencia de los agentes perceptores y sus estados mentales. Una silla se define por ser usada para sentarse, lo cual es una propiedad impuesta por los observadores externos en función de sus intereses o necesidades. De este modo, podemos señalar también otro rasgo de capital relevancia: todo hecho social y todo hecho institucional tienen como base previa un hecho bruto, es decir, todo hecho ontológicamente subjetivo tiene como base un hecho ontológicamente objetivo. 
Detengámonos ahora en qué implica decir que un hecho es epistémicamente objetivo o subjetivo. La subjetividad epistemológica implica que nos movemos en el terreno de la opinión o evaluación personales. Sin embargo, el elemento que resulta más interesante para nuestro análisis es que los hechos ontológicamente subjetivos (esto es, los sociales e institucionales) sean epistémicamente objetivos. Que un hecho sea epistémicamente objetivo implica que no es solo mi opinión, ni es mi mera evaluación: su verdad no depende de la actitud individual de un sujeto. Así, el dinero («este trozo de papel es un billete de cinco euros») o juicios como «Cervantes es el autor de El Quijote» son epistémicamente objetivos.

Es muy interesante para el análisis de lo literario la cuestión de lo que llamaremos defectividad. A menudo un hecho social puede ser defectivo: uno puede intentar fabricar un destornillador por sus propios medios, con tan mala fortuna que ese destornillador no sea capaz de poner ni sacar ningún tornillo; en este caso, estaríamos ante objetos que, creados como hechos sociales, no logran cumplir con la función agentiva para la que han sido construidos. No cumplen por tanto con el primer requisito que habíamos señalado, aunque sí con el segundo; ¿dejan por tanto de ser hechos sociales? Searle se limita a decirnos escuetamente que en estos casos estamos ante hechos sociales defectivos. Así, lo que convierte en hechos sociales a estos objetos defectivos es el hecho de ser vistos, pensados y fabricados como ejemplares de destornillador, aunque de hecho sus propiedades materiales les impidan cumplir la función que los define como tales. La primera condición necesaria pasaría a reformularse no en términos de «poseer las propiedades materiales $\mathrm{X}$ » sino de, o bien tenerlas, o bien ser pensado como si las tuviera.

\section{LOS CONCEPTOS CLAVE II: INTENCIONALIDAD, REGLAS Y FUNCIONES}

Los hechos institucionales son entidades funcionales. Existen para algo. No obstante, la asignación de función es un fenómeno que desborda los límites de los hechos institucionales y que debemos comentar para explicar el conjunto de la propuesta searliana. Pero antes de proceder a esta tarea, consideramos necesario presentar la idea de intencionalidad. La realidad social es creada por la mente, no existiría si no fuéramos capaces de percibir los hechos brutos como algo más que materia. Para que un trozo de papel cuente como dinero o estas manchas como palabras es necesaria una suerte de reconocimiento colectivo al que Searle denomina intencionalidad colectiva (o $\mathrm{We}$ intentionality). Searle define intencionalidad como «aquella propiedad de muchos estados y eventos mentales en virtud de la cual éstos se dirigen a, o son sobre o de, objetos y estados de cosas del mundo» (Searle, 1992: 17). Lo que únicamente a nosotros nos interesa señalar es que la intencionalidad colectiva implica un conjunto de propósitos colectivos sobre los cuales organi- 
zamos nuestras acciones. Además de la intencionalidad, los hechos institucionales requieren la existencia, implícita o explícita, de reglas constitutivas. Searle (1997: 44-47) introduce una distinción capital entre reglas constitutivas y reglas regulativas. Las reglas regulativas «regulan actividades previamente existentes». En cambio, las reglas constitutivas no solo regulan, «sino que crean la posibilidad misma de ciertas actividades»: las reglas del ajedrez crean la posibilidad misma de jugar al ajedrez, que no tiene existencia más allá de esas reglas constitutivas. La afirmación más relevante a este respecto es que todos los hechos institucionales existen solo dentro de sistemas de reglas constitutivas y se crean siempre a través de estas. De este modo, entendemos el concepto de institución como toda realidad resultado de la creación de unas reglas constitutivas.

Toda regla constitutiva se crea mediante una fórmula constitutiva, otro concepto fundamental para entender la ontología social. Y toda fórmula constitutiva tiene la forma

X cuenta como Y en el contexto C (Searle, 1997: 46).

$\mathrm{X}$ corresponde al hecho bruto. Por ejemplo, se conviene arbitrariamente que solo determinado tipo de hecho bruto (papel de tales características, tamaño, etc.) pueda ser Y (dinero) en un contexto C (en la Unión Europea). El término $\mathrm{Y}$ supone por tanto el estatus que pasa a ostentar el hecho bruto, convertido así en hecho institucional. Normalmente esto da lugar a un modo particular de percibir el hecho bruto $(\mathrm{X})$ que genera un concepto nuevo, y entonces ya no vemos trozos de papel, vemos dinero. Es relevante destacar que, en los hechos institucionales, el término Y añade siempre un estatus nuevo, para lo cual el acuerdo colectivo es necesario y suficiente. El término Y, por así decirlo, metamorfosea el objeto ante nuestros ojos.

A esto hay que añadirle el marcador contextual que es el término $\mathrm{C}$, que a su vez implica la intencionalidad colectiva continuada. El término $\mathrm{C}$ engloba otras dos nociones de gran relevancia acuñadas por el filósofo americano, que son lo que llama «network» y «background» (o «red»y «trasfondo»), para cuyo desarrollo nos remitimos al preciso estudio de Rust (2009: 144-153). Se trata además de conceptos que sería fácil vincular con otros propios de la teoría de la literatura como el horizonte de expectativas de Jauss o el habitus de Bourdieu.

Una característica no muy desarrollada, pero que consideramos de suma relevancia, es el carácter iterativo de las fórmulas constitutivas. La iteración supone que lo que es designado como $\mathrm{Y}$ en una fórmula constitutiva puede ser tomado como X para otra nueva fórmula, y así sucesivamente (Rust, 2006: 12; Fotion, 2000: 203). Una vez dicho esto, nos resta únicamente clarificar el papel que juegan las funciones en todo este proceso. Existen dos grandes categorías de funciones: las agentivas y las no agentivas. Ambas son relativas al espectador (Searle, 1997: 33-34), pero mientras las agentivas necesitan para man- 
tenerse una continuada intencionalidad por parte de los agentes, las no agentivas siguen cumpliendo con su función independientemente de nosotros y de nuestros estados mentales. Las funciones no agentivas son exclusivas de los hechos brutos, toda vez que no se imponen a los objetos con propósitos prácticos. El ejemplo al que recurre Searle (1997: 38) es el del corazón, que sirve para bombear sangre (función no agentiva) con independencia de que los agentes lo piensen o no o proyecten o dejen de proyectar sobre el corazón esa función. Esto no implica que no podamos imponerles funciones agentivas a los hechos brutos, como de hecho a menudo hacemos — por ejemplo, si afirmamos que el agua sirve para lavar los platos-.

En este sentido, Searle distingue dos tipos de funciones agentivas: las funciones agentivas causales — que se identificarán con los hechos sociales- y las funciones de estatus — vinculadas a los hechos institucionales_. Ambas son producto de los usos que les damos intencionalmente a los objetos y requieren de la intencionalidad colectiva para existir. En las funciones de estatus, la función se realiza únicamente porque la proyectamos intencionalmente sobre el objeto. De este modo, los hechos institucionales se distinguen de los hechos sociales por la imposición colectiva de funciones a entidades cuyo cumplimiento no radica en su mera estructura física.

Por otro lado, las funciones agentivas causales, análogamente a los hechos sociales, requieren además de esto una causa material en el objeto que lo capacite para desarrollar la función que le es impuesta. La función de sostener la comida para llevársela a la boca - y de ser por tanto un tenedor- requiere para ser cumplida ciertas propiedades en el objeto que son independientes de nuestros estados mentales. Como colofón, resulta interesante comentar el diagrama que Rust (2009: 125) nos presenta:

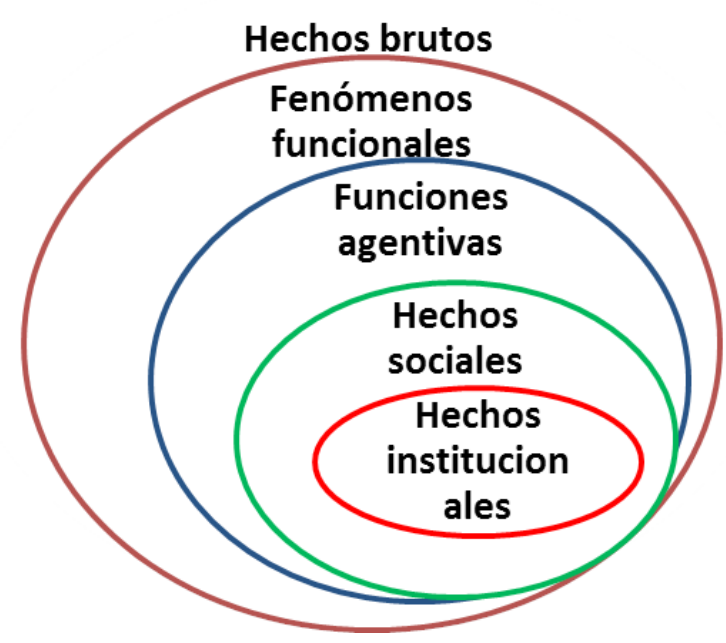


Podemos explicar el cuadro en los siguientes términos:

1. Solo algunos hechos brutos desempeñan funciones. Por ejemplo, el concepto de planeta Júpiter no implica ninguna función, ni agentiva ni no agentiva.

2. Solo algunos de los hechos brutos que desempeñan una función desempeñan una función agentiva. Por ejemplo, la función del corazón de bombear sangre es una función no agentiva.

3. Solo algunos hechos brutos que desempeñan funciones agentivas son hechos sociales. Por ejemplo, si utilizo el agua para lavarme las manos, esta es una función agentiva, pero el agua no por ello es un hecho social.

4. Solo algunos hechos brutos que mediante la imposición de una función agentiva son hechos sociales son hechos institucionales. Por ejemplo, una piedra - hecho bruto - puede convertirse en pisapapeles - hecho social - al asignarle una función agentiva (causal), pero no por ello será un hecho institucional.

5. Algunos hechos brutos sí que se convierten en hechos institucionales al ser investidos de una función agentiva. Por ejemplo, el pedazo de papel que introduzco en una caja de plástico - hecho bruto- cuenta como voto - hecho institucional - al ser investido de una función agentiva de estatus.

\section{HACIA UNA CONCEPCIÓN SEARLEANA DE LA ONTOLOGÍA MUSICAL}

El marco teórico de John Searle guarda una relación directa con algunas propuestas generales en el terreno de la estética. Particularmente, se ha producido en el ámbito de la filosofía de la música una aplicación directa de este andamiaje conceptual, lo que hace fundamental un breve análisis de esta propuesta en nuestro camino hacia el terreno literario.

La cuestión de la ontología y el concepto de música han figurado entre los asuntos más debatidos en la filosofía de la música más reciente (Marrades Millet, 2012: 5-6). Esto ha motivado el intento de algunos críticos de estructurar el campo y las diferentes propuestas coexistentes, si bien consideramos que el marco que parte de John Searle puede ser uno de los que más potencial tenga en este sentido. Stephen Davies (1991) es artífice de una las propuestas más difundidas: en su Definitions of Art distingue dos grandes grupos que denomina functional theories y procedural theories.

Las procedural theories daviesianas (Dickie, 1997: 20) son ni más ni menos que lo que en los últimos años ha solido denominarse definiciones institucionales del arte (García Leal, 2002: 37-92; Dickie, 2000). La expresión teoría institucional, tal y como viene empleándose «preserleanamente», equivale a afirmar que es necesaria algún tipo de institución, circuito de presentación, distribución y valoración de obras artísticas —en tiempos modernos conservatorios o salas de conciertos, por ejemplo- para que podamos hablar de música. Esta acepción no coincide con el sentido que deseamos proponer, se- 
gún el cual una definición institucional es aquella que, independientemente de otros rasgos, se fundamenta en una consideración de la música o de la literatura como hecho institucional. A esto es a lo que en lo sucesivo llamaremos definición institucional, lo cual engloba la primera acepción anteriormente presentada.

Las functional theories establecen que es arte todo aquello que se realiza o concibe con una función X. Sobra decir que en la inmensa mayoría de casos esta función no será otra que la función estética, cuyo máximo abanderado en la filosofía de la música ha sido Jerrold Levinson (2011: 273). El principal problema es que puede argumentarse, como hace Kania (2011), que no toda la música se concibe con un fin estético. ¿Qué finalidad estética cabría aducir en las escalas que todo músico realiza a diario para mejorar sus habilidades técnicas? Presumiblemente ninguna, y no por ello dejan de ser música. La cuestión es que este tipo de definiciones están lejos de la idea de función searleana que antes hemos presentado. Searle nos permite un nuevo desarrollo al incorporar conceptos como los tipos de hechos o la intencionalidad. Además, a la definición funcional estricta, no identificable con el funcionalismo searleano, ha solido achacársele que privilegia ora el lado del creador - del emisor en el esquema clásico de Jakobson-, ora el lado del espectador - del receptor-, lo cual nos remite al problema de lo epistémicamente subjetivo, puesto que un receptor puede reconocer una serie de sonidos como música y otro no y, a menos que fijáramos condiciones necesarias y suficientes, ninguno de los dos se estaría equivocando. Sin embargo, Searle permite solucionar este conflicto con su defensa de las categorías institucionales $-\mathrm{y}$ sociales- epistémicamente objetivadas. Dinero, destornillador, música y literatura coinciden en el modo en que devienen objetivos mediante la intencionalidad colectiva.

\section{UN ESTUDIO PIONERO}

Un reciente artículo sobre el concepto de música, titulado Thinking Clearly About Music y firmado por Vitor Guerreiro (2012), intenta desarrollar parte de lo expuesto en el primer bloque a la reflexión sobre las prácticas artísticas.

Llegados este punto, somos ya capaces de contextualizar la propuesta de Guerreiro, basada en dos referentes fundamentales: de un lado Searle, de otro Dickie (1997) y la distinción entre teorías naturales y teorías culturales. Guerreiro concibe su artículo como un alegato a favor de una ontología de la música inscrita en las coordenadas de las teorías naturales frente a las culturales. Para ello, hace especial énfasis en una teoría natural mejorada con las aportaciones searlianas, particularmente a través de su concepción de los hechos sociales. 
Tras rechazar otros modelos de definición, Guerreiro incorpora varios conceptos de Dickie (1997), en concreto los de actividades naturales y culturales. Las actividades culturales se caracterizan por no estar genéticamente fijadas, a diferencia de las naturales, que sí lo están. Debemos tener en cuenta que muchas actividades culturales no son sino formas en las que se presentan las actividades naturales y que no todas las actividades naturales son biológicamente rígidas - de ahí que puedan darse de distintas formas-. Así, una teoría del arte como actividad natural defenderá que el arte es una actividad natural transcultural independiente de las concepciones del arte que puedan darse en cada momento histórico o en cada cultura. En este punto Guerreiro afirma que su tesis, dirigida claramente hacia la visión de la música que acabamos de enunciar, incluye también su consideración como hecho conceptualmente independiente. Esto es, el hecho de que algo sea o no sea música es independiente del concepto que tengamos sobre ello, es decir, la música es un fenómeno transcultural: los pintores de cuevas primitivos hacían arte, con independencia del concepto institucional que de ello tuvieran. No debemos confundir esta independencia conceptual con el hecho de que la música requiera para existir de la intencionalidad colectiva y de unos determinados estados mentales por parte de los agentes.

Así, es un hecho palmario que han existido y existen distintos conceptos de música —y quizás más aún de literatura—, pero eso no impide la existencia de un concepto de música transcultural independiente de cada concepto institucional que cada sociedad mantenga en cada momento. Los «conceptos culturalmente relativos» (Guerreiro, 2012: 31) son siempre conceptos restrictivos, que, por el motivo que sea, excluyen alguna variante del fenómeno en cuestión. Esto no supone impedimento alguno para que podamos defender la existencia de una definición de música intercultural dentro del marco searleano. Con todo lo dicho podemos reproducir el esquema de Guerreiro, cuyos comentarios, para los que remitimos al propio texto (Guerreiro, 2012: 34-37), iluminan todos los aspectos del cuadro:

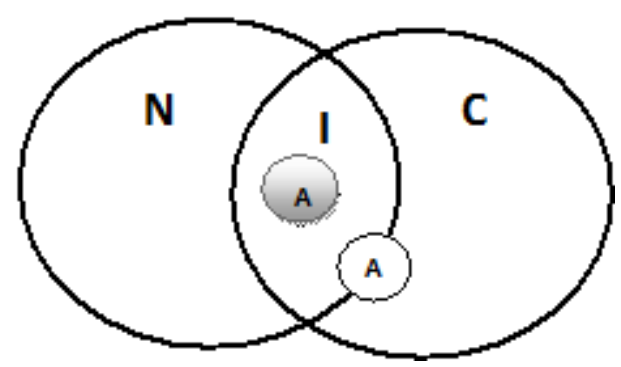

N: Actividades de tipo natural biológicamente rígidas. C: Hechos institucionales creados mediante fórmula constitutiva o actividades culturales. I: Actividades transculturales biológicamente flexibles. AN: Arte según una teoría natural. AC: Arte según una teoría cultural 
Visto este cuadro, la tarea principal de Guerreiro es la de negar que la música pueda incluirse en la categoría $\mathrm{AC}$, esto es, que la música exista en virtud de una función de estatus y sea por tanto únicamente un hecho institucional producto de una actividad cultural. Por ello, concluye su artículo con un modelo de definición que a su juicio se adapta a esta visión de la música como hecho social y AN. Se trata de la definición propuesta por Andrew Kania (2011: 3-13), música es «cualquier evento intencionadamente producido u organizado para ser escuchado, y o bien poseer algún rasgo musical básico como altura o ritmo o bien ser escuchado en virtud de esos rasgos» (Kania, 2011: 12). Si bien puede desarrollarse (Guijarro Lasheras, 2012: 237-252) un comentario más extenso de esta definición, es indudable que se adapta a los rasgos que habría de tener una definición de la música como hecho social.

Lo importante de la definición es que responde claramente al primer requisito de las funciones agentivas causales características de los hechos sociales. Además, el elemento con el que concluye la definición — «o bien ser escuchado en virtud de esos rasgos»-, responde a su vez al segundo requisito de las funciones agentivas causales: el ser percibido, usado, pensado para realizar esa función. Pero Kania va un paso más allá, dado que, en su intento por incluir los casos de extrema vanguardia, admite la posibilidad de que algo pueda ser música aun en la ausencia total del primer requisito antes señalado, bastando con ser escuchado como si lo cumpliera. Kania adopta por tanto una posición inclusiva ante la defectividad. Son estas ideas las que nos darán la llave para desarrollar la reflexión sobre el hecho literario.

\section{Algunos Aspectos Generales}

En los siguientes apartados se pretende mostrar algunas vías a través de las cuales trasladar lo expuesto hasta ahora a la teoría literaria. Como se verá, las posibilidades desbordan los límites de este trabajo, de forma que muchas quedarán únicamente esbozadas. Libros recientes como el último y brillante libro de Terry Eagleton (2013), centrado en exclusiva en la pregunta de qué es literatura, muestran que estamos ante una cuestión que sigue despertando un sostenido interés.

Un elemento que la perspectiva searlena nos permite identificar de base es tanto lo que llamaremos la falacia etimológica como el rechazo a las definiciones que aun siendo verdaderas no singularizan ni explican el objeto definido (Llovet et al., 2005: 32, por ejemplo). La realidad social e institucional se genera y mantiene con total independencia de los significantes que elijamos para designarla — de hecho en ocasiones ni siquiera contamos con un significante- - Por esto, no es necesario aquí acometer una definición de literatura presentando un listado de acepciones del término, como podría hacerse desde un enfoque onomasiológico (Garrido Gallardo, 2004: 19-22). 
Compartimos pues propósito con Richard Ohmann (1999: 11), «dese[amos] una definición que proporcione un adentramiento en la naturaleza de la literatura, en lugar de una simple información sobre el sentido de la palabra literatura en su uso generalizado». A muchos, el mero empleo del vocablo «naturaleza» les generará reticencias. Pero esta tensión se soluciona al advertir que esta naturaleza no ha de ser necesariamente un conjunto de rasgos - de hechos brutos - presentes en lo literario, sino que puede ser, parcial o totalmente, un conjunto de hechos institucionales y funciones de estatus.

$\mathrm{Y}$ es que, si una cosa parece clara, es que la literatura no es un hecho bruto. La literatura no es un hecho que se dé con independencia de los seres humanos, sus actividades, intenciones y estados intencionales. Al contrario, requiere de estos para existir. Esto tiene una relevante implicación: es taxativamente imposible ofrecer una definición de literatura basada únicamente en rasgos intrínsecos a los objetos que llamamos literarios. Si no, esta pertenecería a la misma categoría que las piedras, el agua o las montañas. Ahora bien, cometeríamos un error si de aquí dedujéramos que debemos necesariamente prescindir de cualquier caracterización intrínseca del objeto literario. Es también reseñable que detrás de todo hecho social e institucional hay siempre un hecho bruto. Y así ocurre en la literatura: unas manchas de tinta, una serie de ondas acústicas, una serie de bits de información encriptados en un circuito informático son los hechos brutos más frecuentes a partir de los cuales se crea la literatura, si bien estos hechos brutos base no sirven para esclarecer su naturaleza ontológicamente subjetiva.

Searle nos previene así de caer en el peligro de lo que García Leal (2002: 23) llama el apriorismo, «o sea, introducir definiciones de arte que sean previas y para nada tengan en cuenta la realidad efectiva de las prácticas artísticas». Reformulando la cuestión, el peligro del apriorismo no es otro que el de tratar a la literatura como si fuera un hecho bruto ontológicamente objetivo. Así, cuando a menudo se afirma que la literatura no tiene una «naturaleza», lo único que se está queriendo decir es que no es un hecho bruto.

Al ser necesariamente un hecho social o institucional, la literatura es ontológicamente subjetiva: su existencia depende de nuestros estados mentales. Y es también epistémicamente objetiva. Es un hecho epistémicamente objetivo que en nuestra sociedad acordamos ver el Quijote como obra literaria: no depende de mis creencias o actitudes al respecto. La extensión del concepto de literatura está objetivada por la intencionalidad colectiva que acuerda ver determinados textos como literarios. Hay por tanto un consenso sobre lo que cuenta como literario aun en el disenso. De este modo, una de las preguntas más interesantes a las que debería responder nuestra propuesta es por qué objetivamos epistémicamente determinados textos como literatura y otros no, y qué principios rigen la objetivación de la literatura. Podemos preguntarnos si, cuando se afirma «el Quijote es literatura», se está haciendo una afirmación dependiente o independiente del observador. Esto es, debemos hallar una 
respuesta a si este aserto pertenece a la misma categoría que, por ejemplo, «mi coche es rápido»-dependiente del observador-, o pertenece a la de «mi coche pesa 870 kilos» —independiente del observador- En el fondo, esto no es sino plantearse si la literatura es un hecho institucional o social respectivamente.

Esto nos lleva a examinar la cuestión de si, como afirma Eugenio Trías (1998: 207) puede haber «falsos pretendientes» a la categoría de literatura. Desde un punto de vista institucional, podríamos hablar de billetes falsos: algo pretende hacerse pasar por la $\mathrm{X}$ de la fórmula constitutiva para así poder ser $Y$, pero en realidad no cumple los requisitos para ser X. Así, podríamos determinar qué rasgos, en una cultura y en un momento determinados, se exigen para que algo sea visto como literatura - por ejemplo, en determinado momento podría ser condición necesaria el poseer un carácter ficcional-, y podríamos detectar casos de textos que se asemejan, que podrían pasar en ese contexto dado por textos ficcionales, pero que en realidad no lo fueran - unas memorias de un superviviente de Auschwitz, por ejemplo- Ese sería un caso de defectividad institucional, de un texto que se parece a lo que llamamos literatura pero que no lo es, aunque pudiera serlo en el momento mismo en que variara esa fórmula constitutiva.

Desde un punto de vista de la literatura como hecho social, la defectividad es algo ligeramente distinto. Un texto literario defectivo sería todo aquel que no cumpliera con los rasgos intrínsecos necesarios — que no suficientespara ser literatura, aunque de hecho pareciera poseerlos. Así, por ejemplo, todo aquel texto en el que no detectáramos un uso literario del lenguaje —en el supuesto de que tal cosa exista - aunque hubiera sido escrito con ese fin, sería un caso de defectividad social, análogo al de la bombilla con el filamento roto que no puede dar luz. La cuestión de la defectividad nos permite ya perfilar dos opciones teóricas claramente delimitadas: por un lado podemos pensar que la literatura es un hecho social, por otro, como muchas teorías a lo largo del siglo $\mathrm{XX}$, que se requiere un acercamiento únicamente institucional para explicar el fenómeno literario. Los siguientes apartados tratarán de desarrollar estas dos ideas.

\section{LITERATURA Y HECHOS SOCIALES}

Algunas propuestas generales, así como algunos aspectos particulares de algunas teorías de las que han tenido lugar a lo largo del siglo XX, pueden comprenderse mejor a la luz de su explicación como teorías sociales de la literatura. Recordemos que, básicamente, una visión de la literatura como hecho social debe fundamentarse en dos pilares básicos: que hay una serie de textos que llamamos literarios porque (1) tienen una serie de rasgos intrínsecos que los capacitan para ser literarios y (2) son colectivamente representa- 
dos, vistos, producidos o leídos como literarios. La literatura sería así un hecho análogo a los sacacorchos. A estos dos puntos nos referiremos en lo sucesivo como primer y segundo requisito. La primera tarea por tanto de una teoría social sería la de esclarecer qué rasgos son necesarios pero no suficientes para que se cumpla la función agentiva causal correspondiente y pueda existir la literatura (requisito 1). Lo más interesante es que podemos reinterpretar buena parte de la investigación teórica llevada a cabo en el siglo XX como un intento de ello, empezando por el movimiento que más impulsó la teoría literaria en el pasado siglo, el formalismo ruso, así como todas las teorías acerca de la literariedad o el lenguaje literario, que pueden contextualizarse histórica y pluralmente en Garrido, Todorov et al. (1987).

En primer lugar, es evidente que, sea lo que sea aquello en lo que hagamos recaer los rasgos distintivos del lenguaje literario, habrá textos que posean esas propiedades y que no sean literarios. Esto no nos preocupa tanto porque podrá argumentarse que no lo son simplemente porque no cumplen el segundo requisito. La pregunta que podemos hacernos, declinando entrar en la cuestión de la existencia o inexistencia del lenguaje literario o de un uso literario del lenguaje, es si este es una potencialidad derivada de las propiedades de determinados textos o es tan solo producto de la intencionalidad humana proyectada hacia el lenguaje - lo que equivaldría a decir que el lenguaje literario no existe como tal-.

Lo mismo sucede con el tan comentado concepto de literariedad, entendido como el conjunto de rasgos que nos permiten ver una obra como literaria, aunque estar en posesión de todos esos rasgos no haga que necesariamente el texto en cuestión sea literatura. No concebimos por tanto la literariedad simplemente como «acción y efecto de ser literatura», lo que a nuestro juicio haría perder el interés y potencial explicativo al concepto. El objetivo principal del formalismo no fue el de hallar la clave de qué es literatura sino mostrar qué debe tener un texto para poseer literariedad, lo cual no lo convierte necesariamente en literatura: el propio Jakobson afirma que «el objeto de la ciencia literaria no es la literatura sino la literariedad» (Bobes Naves, 2008: 212). Esto es, sus estudios deben centrarse en el primer requisito, no en el segundo. Jakobson demuestra así ser consciente de que la categoría literatura desborda los márgenes de lo estrictamente textual.

Algunos teóricos de la literariedad, como Tynianov y Hockett, afirman que la literariedad es una «connotación sociocultural variable» (Lázaro Carreter, 1999: 153). La cuestión, en el marco searliano, vendría a reformularse del siguiente modo: ¿es esa literariedad que buscamos un rasgo ontológicamente objetivo del objeto literario o es ontológicamente subjetivo pero epistémicamente objetivo? No es nuestro propósito aquí ahondar en este particular, si bien parece más factible lo segundo, que es exactamente lo que señalan Tynianov y Hockett. Esto implica que la literariedad no sería una propiedad material inmutable de un texto — como sí que lo sería la posibilidad de sacar corchos 
en el sacacorchos-, sino derivada de diversos constructos culturales estables pero sujetos a cambio - el predominio de la connotación, la capacidad desautomatizadora, por ejemplo- Sería por tanto epistémicamente objetivo que en un determinado texto existe un predominio de la connotación o una voluntad y capacidad desautomatizadora, pero solo en un marco cultural dado. Así, podríamos decir que Madame Bovary posee literariedad del mismo modo que un Ferrari es un coche rápido. Ambas serían epistémicamente objetivas y se basarían en algo más que el mero capricho y consenso de los agentes. Ahora bien, así como un Ferrari de los años 40 ya no puede considerarse un coche rápido, la literariedad de una obra podrá variar contextual y temporalmente.

Así, esa finalidad de, por ejemplo, desautomatizar la percepción de la realidad — nótese el componente teleológico y funcional—, sería el primer requisito de los hechos sociales y las funciones agentivas causales. Es cierto que esta potencialidad de desautomatizar es relativa al observador (Searle, 1997: 34), lo cual no implica que toda función sea válida y posible en todo momento ni epistémicamente subjetiva o conceptualmente dependiente. Es justamente esto lo que cabría replicarle a pragmatistas como Richard Rorty desde la perspectiva social searlena. La visión de Rorty (Eco, 2002: 120), quien elige curiosamente el ejemplo del destornillador, implica, lo pretenda o no, una particular versión de la teoría institucional de la literatura.

Puede defenderse entonces que la percepción de un objeto determinado como destornillador o como literatura responde a una selección de determinados «rasgos pertinentes». Esta selección podrá ser susceptible de cambio: en una sociedad en la que los objetos a los que llamamos destornilladores solo se concibieran como arma de defensa personal, estos objetos no serían destornilladores. Por eso, el hecho de poder usar un destornillador para rascarse la oreja o El Quijote para analizar la construcción de los roles de género en el siglo XVII no modifica la intencionalidad colectiva que recae sobre esos objetos ni los rasgos que el propio objeto posibilita tomar como pertinentes.

En otro orden de cosas, podemos interpretar una corriente tan relevante como la estética de la recepción como la toma de consciencia de la insuficiencia del primer requisito y el intento de desarrollar el segundo punto de los hechos sociales, toda vez que su principal reivindicación es la de la necesidad de tener en cuenta el papel y respuesta del lector en la obra literaria. En su versión más extrema, la estética de la recepción podría ser una teoría institucional de la literatura, siempre y cuando depositara en los lectores la competencia exclusiva, no ya de cerrar la obra literaria, sino de crearla en su integridad, siendo las comunidades de lectores las que generan el marco en el que poder hablar de literatura, lo cual remite a las procedural theories antes mencionadas.

Pero no quisiéramos continuar este análisis sin antes detenernos en una atractiva reformulación del concepto de literariedad. Se trata de la literariedad como parecido familiar, noción acuñada por Wittgenstein y muy popula- 
rizada en la filosofía del lenguaje. Es muy interesante mencionar que el propio Searle (Eagleton, 2013: 41) afirmaba en los años 70 que la literatura «es un concepto basado en el parecido familiar». Para explicar en qué consiste esto, nadie mejor que el propio Eagleton:

En un pasaje célebre, Wittgenstein nos invita a reflexionar sobre qué es lo que tienen en común todos los juegos, y concluye que no comparten ni un solo elemento. Lo que más bien hay es «una compleja red de parecidos que se superponen y entrecruzan». A continuación, hace la famosa comparación de esa enmarañada red de afinidades con los parecidos existentes entre los miembros de una familia. Quizás esos hombres, mujeres y niños se parezcan, pero no porque todos tengan las orejas peludas, la nariz muy prominente, una boca muy salivosa o cierto toque de petulancia. Algunos tendrán uno o dos de estos rasgos, pero no más, otros tendrán una mezcla de varios junto con, acaso, alguna característica física. De esto se desprende que tal vez dos miembros de la misma familia no tengan en común ninguna en absoluto (Eagleton, 2013: 40-41).

Esto implica que no es posible hablar de condiciones, no ya necesarias-ysuficientes, sino tampoco necesarias ni suficientes. Así pues la literariedad — palabra que el propio Eagleton (2013: 47) llega a emplear- pasa a entenderse como un conjunto difuso de rasgos ni necesarios ni suficientes que para el teórico británico son además enumerables: cuando llamamos literario a algo queremos hacer notar alguna de estas cinco características o una combinación de ellas:

una obra que sea de ficción, arroje intuiciones significativas sobre la experiencia humana (a diferencia de informar sobre verdades empíricas), utilice el lenguaje de un modo especialmente realzado, figurativo o deliberado, no tenga utilidad práctica en el sentido que lo tiene una lista de la compra o constituya un texto muy valorado (Eagleton, 2013: 46).

Como vemos, la propuesta sigue encajando plenamente dentro de los hechos sociales, a la que restaría añadirle algún tipo de versión intencional de la estética de la recepción para completar así los dos requisitos necesarios.

Ahora bien, las propiedades de la literatura una vez acontecida y, por tanto, su definición como hecho social, no son ninguna invención y son independientes de nuestras creencias sobre ellas, que no de la intencionalidad que proyectamos sobre ellas. Del mismo modo, una cultura puede concebir y definir el matrimonio como sacramento divino, lo cual no altera en absoluto los rasgos, funciones y reglas constitutivas de la institución del matrimonio. Esto nos lleva a un punto de gran relevancia: la posibilidad de plantear una definición de la literatura como hecho social transcultural —esto es, como hecho social y actividad natural biológicamente flexible, según lo expuesto en los capítulos anteriores de este trabajo- - Una de las críticas más frecuentes ante la idea de un concepto transcultural de literatura es la incuestionable mutabilidad que experimenta. Lo que en una civilización es literatura resulta no serlo en otra. Lo que en un tiempo se considera literatura, queda fuera de lo literario en otro. 
Pero esta mutabilidad no impide el tipo de definición que nos planteamos, ya que tiene lugar debido a los diversos conceptos institucionales de literatura vigentes en cada cultura: no existe así una continuidad entre las diversas definiciones institucionales — que pueden ser completamente erróneas- y una hipotética definición social de la literatura.

Visto esto, la idea de una definición social y transcultural de literatura salva por tanto estos escollos. Así, habiendo también redefinido el concepto de literariedad, y tomando como modelo la definición musical de Kania expuesta en el capítulo sexto, podríamos decir que una visión social pasa por entender la literatura como cualquier texto intencionadamente producido $\mathrm{u}$ organizado para poseer literariedad, y, o bien poseer algún rasgo (literario) como carácter ficcional, moral, lingüístico, no pragmático o normativo, o bien ser leído en virtud de esos rasgos. Es vital señalar que los cinco «rasgos literarios» mencionados son los cinco especificados por Eagleton (2013: 46) ya comentados como rasgos de parecido familiar de la literatura. Sin duda, cabría replantearlos, añadir o suprimir alguno sin que esta propuesta de definición se alterara o resintiera por ello.

Esta aproximación social a la literatura permite, como la de Kania, incluir los llamados casos extremos, esto es, los ejemplos de vanguardismo más rabioso que tantos problemas plantean al teórico. Imaginemos (1) un poema que sea una página en blanco y (2) una receta de cocina estándar presentada, editada y publicada — en una editorial literaria cuya colección se llamara «Última Narrativa», por ejemplo- como obra literaria. Es evidente que estas obras no poseerían ninguno de los rasgos arriba especificados, así como tampoco ninguno que cupiera identificar con la literariedad. Sin embargo, están producidos y organizados para poseer literariedad — aunque no la tengan-, y la manera que tiene el autor de indicárnoslo es presentándola dentro del marco artístico que hace a los lectores leer esos textos «en virtud de esos rasgos», aunque, repetimos, en realidad no los posean. Reafirmamos aquí por tanto la idea que Kania (2010) defiende en su esclarecedor artículo sobre la música sin atributos. Parafraseándolo, podemos concluir que puede haber poemas en blanco en una tradición poética incluso cuando ninguna tradición poética pueda estar íntegramente constituida por poemas en blanco, lo cual no entra en conflicto con una visión de la literatura como hecho social.

Una última cuestión derivada de todo esto, de la que merece la pena ocuparse, es la que plantea Umberto Eco (1971) en su libro precisamente titulado La definición del arte. Eco muestra la caducidad de las formas artísticas y se centra en la muerte del arte que muchos profetizaban en la década de los 60 fruto del advenimiento de las formas más radicales de vanguardismo (Eco, 1971: 149). Así, el semiólogo italiano constata que unas formas artísticas mueren y surgen otras que no poseen ni un solo rasgo en común con las anteriores. Esto, como vemos, no supone impedimento alguno para lograr una definición social de la literatura. Podemos, a efectos de clarificarlo lo más posible, 
trazar una analogía con un hecho social como los relojes. En efecto, no hay ningún elemento común entre un reloj de sol, un reloj analógico y un reloj digital, pero esta transformación histórica no supone ningún impedimento para hablar de que, por supuesto, existe un tipo de hechos sociales a los que llamamos relojes y los tres tipos antedichos lo son. No tiene por tanto sentido hablar de una muerte del arte derivada, en este caso, de su autoconsciencia, de la primacía de la idea en detrimento de la obra (Eco, 1971: 129). Sí, en cambio, podemos defender la muerte de un determinado concepto institucional del arte, o su ruptura, lo cual no afecta al hecho social que es la literatura.

\section{LITERATURA Y HECHOS INSTITUCIONALES}

Buena parte de las teorías literarias más relevantes de la segunda mitad del siglo XX pueden entenderse como diferentes versiones de una visión de la literatura como hecho institucional. En su enunciación más general, una teoría institucional de la literatura sería toda aquella que afirmara que para que un texto sea literario lo único necesario es que sea representado colectivamente como tal. Esto es, que no hay una esencia o naturaleza de lo literario como fenómeno propiamente textual, lo cual implica también la inexistencia de cualquier cosa susceptible de ser llamada lenguaje literario. O, en el mejor de los casos, su irrelevancia: que exista o no exista el lenguaje literario no nos sirve en ningún punto para determinar qué es literatura y por qué unos textos ostentan el estatus de literarios y otros no.

La literatura sería así un fenómeno análogo al dinero, los presidentes, fronteras o matrimonios. En el momento en el que unas reglas constitutivas compuestas de fórmulas constitutivas, implícitas o explícitas, hicieran percibir colectivamente a los agentes determinados textos como literarios, existiría la literatura, con independencia de las propiedades de esos textos. Ahora bien, intentaremos mostrar que esta visión de la literatura admite numerosos matices que pueden desembocar en visiones bastante diferentes del fenómeno literario.

Podemos establecer desde el primer momento algunos elementos más como base de toda teoría institucional. Con carácter general conviene recuperar la idea de que la estructura de las instituciones humanas es una estructura de reglas constitutivas. También, que la gente que participa en las instituciones no es normalmente consciente de esas reglas; a menudo tienen incluso creencias falsas sobre la naturaleza de la institución, y hasta a las mismas personas que crearon la institución puede pasarles por alto y desapercibida su estructura (Searle, 1997: 139). Así pues, una definición institucional no deberá intentar delimitar cuáles son las creencias que en un momento determinado una comunidad tiene respecto de la literatura. Esta es, desde luego, completamente mutable y relativa. En contraposición - y este será el componente que per- 
mita proponer una definición institucional transcultural-, puede defenderse que las reglas constitutivas - y la función- del fenómeno literario son esencialmente las mismas para todo aquello que se llama o ha llamado literatura, del mismo modo que es común a todo lo que ha sido alguna vez dinero el ser visto como medio de pago e intercambio. Por contra, también cabría argumentar que estas reglas constitutivas son particulares de cada sociedad y tiempo histórico, sin posibilidad de hallar un punto de encuentro común en un plano superior, lo cual nos lleva a una definición institucional relativista de la literatura.

Para analizar todo hecho institucional, debemos atender a tres elementos: su creación, su existencia continuada y su representación oficial —normalmente lingüística- (Searle, 1997: 126). Uno de los elementos fundamentales que posibilitan la creación de la literatura es su carácter iterativo. Así, tenemos que un conjunto de manchas de tinta en un papel $(\mathrm{X})$ cuentan como signos, como palabras (Y) en el idioma castellano (C). Estas palabras (X) cuentan como texto (Y) al ser agrupadas o presentadas conjuntamente de determinada forma (C). A su vez, este texto $(\mathrm{X})$ cuenta como literatura $(\mathrm{Y})$ en, por ejemplo, la sociedad occidental del siglo XXI (C). El marcador contextual (C) es siempre necesario en toda fórmula constitutiva, lo cual supone que el conjunto de hechos brutos $-\mathrm{o}$ hechos sociales o institucionales que, en su primer nivel, son siempre hechos brutos- que actúen como base X para obtener el estatus de literatura es siempre un conjunto convencionalmente delimitado. El conjunto de textos a los que llamamos literatura podría ser perfectamente distinto del que es si la fórmula cambiara. Esta visión tiene la ventaja de que explica satisfactoriamente la mutabilidad de lo literario, es decir, el hecho de que en unas épocas un texto sea considerado literatura pero en otras no.

Habíamos argumentado que la $\mathrm{Y}$ de la fórmula tiene que rebautizar al hecho bruto $\mathrm{X}$ en que se basa, tiene que investirlo de un estatus inexistente con anterioridad - dinero, matrimonio, frontera, literatura-. La cuestión que debemos plantearnos por tanto es en qué consiste este nuevo estatus literario aplicado a un texto, de qué forma nos hace alterar nuestra percepción, lectura o aproximación al mismo. Esto nos podría conducir a un concepto de literariedad intencional que el lector proyecta sobre la literatura pero que en ningún caso es una propiedad formal del texto. Así, presta más atención al uso del lenguaje, intenta obtener placer estético, suprime el interés práctico, potencia el aspecto connotativo del texto, etc. Todos estos rasgos serían a la literatura lo que servir como intercambio de bienes al dinero o separar naciones y territorios a las fronteras.

De todo esto se desprende que tan acertado es que en una época se considere literario un texto como que en otra no. Quizás solo cupiera puntualizar a este respecto que hay textos que se prestan más por sus rasgos intrínsecos a pasar a formar parte de la definición institucional de la literatura y de la función de estatus correspondiente, del mismo modo que un pedazo de níquel de 
dos centímetros de diámetro se presta mejor a servir como dinero que un elefante.

Esto nos lleva a establecer un concepto que consideramos clave y al que llamaremos la apertura bruta de los hechos institucionales. Un hecho institucional tendrá mayor apertura bruta cuantos más hechos brutos sean susceptibles de ser tomados como X para la fórmula constitutiva que genera el hecho institucional Y en cuestión. Así, la apertura bruta de una función agentiva como la de ser presidente se circunscribe a los seres humanos. En el caso del dinero, la apertura bruta es bastante mayor: tan solo se requiere ser un material almacenable e intercambiable. Aun así, hay algunos hechos brutos que no podrían ser nunca dinero, por ejemplo, la luna o el planeta Júpiter. La literatura tiene un grado intermedio de apertura bruta: es mayor que, por ejemplo, la de la toma de posesión de un presidente, pero es menor que, por ejemplo, la del dinero. La literariedad puede entenderse desde esta perspectiva institucional como la delimitación de esta apertura. En consecuencia, es posible hallar una definición global de literatura, igual que es posible hacerlo del dinero, pero no de los textos que son o no literatura, ya que para identificar esto habrá que desenterrar las reglas constitutivas implícitas de cada momento y sociedad, que serán por necesidad diferentes entre sí, variables y relativas. Esto quiere decir que el término $\mathrm{Y}$ de la fórmula constitutiva es definible con independencia de los $\mathrm{X}$ que lo encarnen.

Además de ser creado, el estatus de ser literario — la literatura - ha de ser mantenido y reconocido. Esta perspectiva supone otra vía interesante para los estudios literarios. Para que algo sea literatura «un número suficiente de miembros de la comunidad relevante deben seguir reconociendo y aceptando la existencia de esos hechos» (Searle, 1997: 128). Por eso, nos dice Searle, la fórmula constitutiva tiene en la misma medida la función de la creación como la del mantenimiento del hecho social. Un elemento fundamental para este último es lo que el filósofo americano denomina indicadores de estatus (Searle, 1997: 130-131): la firma en los contratos o el uniforme de un policía nos sirven para identificar un hecho institucional ya construido. Una tarea del teórico literario será por tanto la de localizar los distintos indicadores de estatus que permiten o contribuyen a que percibamos un texto como literario. Algunos de ellos, en nuestro tiempo, son sin duda las distintas editoriales, la ubicación del texto en un determinado lugar de la librería, los paratextos - las inscripciones de la portada y contraportada, por ejemplo- Esto no quiere decir que no pueda haber falsos indicadores: puedo ponerme un uniforme de policía y no por eso soy policía, o puedo publicar un manual de principios de termodinámica bajo el rótulo «Narrativas Hispánicas» sin que eso convierta institucionalmente al texto en literatura.

Una vez analizado esto, debemos ocuparnos de una noción insoslayable en la visión institucional de la literatura: las funciones de estatus. Sea más o menos lábil, es fácil identificar la función de estatus que cumplen elementos 
como el dinero o los presidentes. Pero en el caso de la literatura la cosa se complica: no existe una función o funciones tan evidentes, a menos que recurramos, como hacía Levinson para la música, a una función estética. De todas maneras, la evidencia de que puedan detectarse otras funciones no altera este estado de cosas, análogamente al hecho de que podemos decir que el lenguaje sirve para comunicar estados mentales, si bien esto no alcanza a explicar la función de estatus que desempeña. Nos parece de todas maneras esencial distinguir entre función y utilidad desde el pragmatismo searliano. La función no tiene por qué conllevar un fin práctico, al contrario que la utilidad —el usoque le damos a las cosas. Son dos cuestiones netamente separadas: responder a la pregunta de la función de la literatura no tiene nada que ver con determinar por qué consideramos o escribimos un texto literario: por dinero, búsqueda de reconocimiento, prestigio, placer estético... La lista puede ser interminable. La función será una proyección humana sobre un texto que nos haga percibirlo o leerlo de forma distinta al modo en que lo percibiríamos o leeríamos si no le confiriéramos el estatus de literatura. Por ello, el propio Searle se rebela contra la idea de que el suyo constituya ningún tipo de «análisis funcional o de explicación funcional» (Searle, 1997: 126) de los hechos institucionales. El concepto de función de estatus se relaciona de manera interna con el estatus correspondiente, esto es, el estatus «implica trivialmente la función correspondiente». Searle matiza a su vez que:

El caso es que la intencionalidad colectiva impone un estatus especial a algún fenómeno, y con ese status, una función. [...] Algunas veces la función en cuestión solo de un modo muy general está determinada o implicada por la expresión de status, y, a veces, lo implicado es un abanico entero de funciones, más que una singular función específica (Searle, 1997: 125-126).

Está claro que la literatura pertenece a este segundo tipo. El concepto de utilidad, en cambio, supondría una relación ajena al propio estatus de literatura. Por eso, es capital apuntar que todo lo señalado por Eagleton en un pasaje como el que sigue cae completamente fuera de la idea searliana de función:

Toda definición funcional del arte tendrá en cuenta de manera natural la sobrecogedora diversidad de sus usos y efectos. Algunas cosas, como las cucharas o los sacacorchos, se pueden definir con facilidad apelando a su función, que históricamente hablando no ha dejado de ser bastante estable. Pero por sus funciones la literatura ha tenido una historia mucho más accidentada, que abarca desde consolidar el poder político o cantar las alabanzas del Todopoderoso hasta ofrecer instrucción moral o dar muestras de imaginación trascendental (Eagleton, 2013: 45).

Eagleton no solo está mezclando hechos sociales — cucharas y, curiosamente, de nuevo sacacorchos - con lo que él mismo considera un hecho institucional — la literatura_, sino que baraja indistintamente las funciones agentivas potencialmente definitorias del fenómeno — toda vez que guardan una 
relación interna con el estatus en cuestión- con las diversas utilidades que se le puede dar a la literatura. En relación a esto debemos añadir otra distinción: no debemos confundir las funciones agentivas - recordemos que las funciones de estatus son un subtipo de funciones agentivas - con las no agentivas (Searle, 1997: 40). Todos los hechos institucionales desempeñan, simultáneamente a las funciones de estatus que les confieren su institucionalidad, multitud de funciones no agentivas, esto es, funciones que se dan con independencia de que los agentes las piensen, la intencionalidad que proyecten o cualquier otro rasgo sinónimo. Por ejemplo, el dinero desempeña la función de mantener los privilegios de las clases sociales dominantes; el matrimonio controla socialmente la reproducción y la conducta sexual de los individuos. La literatura desempeña la función de perpetuar o subvertir la ideología socialmente aceptada — «consolidar el poder político», dice Eagleton-, o de dar de comer a los filólogos. Todas ellas son funciones no agentivas que no tienen ningún papel en la construcción del hecho institucional y que están siempre sujetas a cambio.

Estas ideas permiten explicar la mutabilidad de lo literario - la consideración de un texto como literario o no en diversos momentos y comunidadescomo un caso de reasignación de función y de estatus, tanto en un eje diacrónico — una misma comunidad a lo largo del tiempo- como sincrónico — dos comunidades distintas en un momento dado-. Así, en distintas comunidades (dado un $\mathrm{C}$ distinto), el mismo hecho bruto $\mathrm{X}$, puede estar vinculado con otros Y, ostentando un estatus diferente (Rust, 2009: 120), lo cual no entraña contradicción alguna. Además, esto supondría la muerte del autor no ya a un nivel hermenéutico sino también epistémico: el autor no tiene ninguna capacidad para fijar o imponer la condición de literario o de no literario a un texto. De lo contrario, un texto concebido como documento informativo (una crónica de un viaje, por ejemplo) nunca podría pasar a ser visto como literario, ni viceversa. Esto nos lleva a plantear el problema de la subjetividad epistemológica de lo literario: toda teoría institucional debe por tanto poder explicar por qué existen desacuerdos sobre el carácter literario de un texto dentro de una misma comunidad lectora. El vanguardismo puede explicarse también desde este planteamiento: supone un cambio $-\mathrm{O}$ un intento de cambio- de la formula constitutiva vigente.

Llegados a este punto, podemos plantear como mínimo tres tipos de definición institucional de literatura. En primer lugar existen (1) definiciones institucionalizadas. Son siempre, recuperando la idea de Dicke (1997 y 2000), definiciones enmarcadas: toda aquella definición epistémicamente objetivada que se da en un marco determinado. Son por tanto definiciones siempre contextuales y sujetas a cambio, que responden a la idea de que no puede haber literatura fuera de un marco que sancione algo como literario. Y pueden ser erróneas, esto es, no reflejar las reglas constitutivas que verdaderamente están posibilitando su existencia. Los ejemplos pueden ser bastante esclarecedo- 
res: una cultura puede definir literatura como el conjunto de textos inspirados por el furor platónico e inspiración divina actuante a través del poeta. Esta sería una definición institucionalizada que no daría cuenta del estatus (Y) merced al cual un texto se convierte en literario.

Junto a estas definiciones institucionales contextuales, tenemos también (2) definiciones institucionales propias. Son todas aquellas producto de considerar la literatura un hecho institucional. Suelen tratar de explicar la fórmula constitutiva que hace posible otorgar el estatuto de literatura a un texto. Un tipo particular serían las (3) definiciones institucionales transculturales, centradas en el elemento $\mathrm{Y}$ de la fórmula constitutiva. Su singularidad consiste en defender que el considerar a algo como literario supone una serie de actitudes comunes en todo momento y época hacia los textos, aunque estos varíen temporal y geográficamente, así como las creencias sobre lo literario. En consecuencia, las distintas definiciones institucionalizadas (1) se entienden como versiones «restrictivas» (Guerreiro, 2012: 31) de una posible definición transcultural (3). Una definición institucional transcultural del dinero sería del tipo «todo aquello que sirve como intercambio simbólico de bienes», o algo similar. Una tarea de la teoría de la literatura sería entonces esclarecer tanto unos tipos como otros de definición. Es evidente que, desde cualquiera de estos puntos de vista, es literatura lo que acordemos ver como literatura. Stanley Fish escribe al respecto:

No es que la literatura exhiba ciertas propiedades formales que exigen un cierto tipo de atención; más bien, prestar un cierto tipo de atención (definida por lo que se entiende que haya de ser literatura) tiene como resultado que se advierten propiedades que sabíamos por adelantado que eran literarias (García Leal, 2002: 98).

Lo único que está haciendo Fish es reaccionar contra una visión social y abogar por un acercamiento canónicamente institucional a la literatura. Eagleton (2013: 64), por su parte, nos permite traducir la propuesta de Fish y afirmar que interpretar es crear hechos institucionales: es ver determinados textos como literatura. La interpretación genera el hecho institucional, y no al revés.

Precisamente por eso, el hecho de que haya textos literarios que no compartan ni una sola propiedad no puede considerarse impedimento para una definición institucional transcultural: tampoco comparten propiedades un billete de cinco euros, un dinar romano y una ficha de plástico de casino y todos ellos son dinero. Es precisamente Eagleton, uno de los críticos con mayor visibilidad de los últimos tiempos, quien ha sostenido una tesis plenamente identificable con las visiones institucionales que en este capítulo exponemos. En uno de sus estudios más conocidos, el crítico marxista constata la mutabilidad y labilidad de lo literario. Es imposible señalar un conjunto de características intrínsecas de todo lo que se ha llamado literatura, a la vez que «si la gente dice que tal o cual escrito es literatura, parecería que de hecho lo es» (Eagleton, 1988: 19-21). Pero Eagleton introduce un componente marca de la casa: 


\begin{abstract}
Si no se puede considerar la literatura como categoría descriptiva «objetiva», tampoco puede decirse que la literatura no pasa de ser lo que la gente caprichosamente decide llamar literatura. Dichos juicios de valor no tienen nada de caprichosos. Tienen raíces en hondas estructuras de persuasión al parecer tan inconmovibles como el edificio Empire State (Eagleton, 1988: 28).
\end{abstract}

La percepción de un texto como literario se relaciona estrechamente con las ideologías sociales. La literariedad es producto de una determinada visión del mundo — una ideología - que se da de distinta forma según la clase social a la que pertenezcan los sujetos. Es más, la ideologización que implica el fenómeno institucional puede considerarse inevitable en tanto que constitutiva del propio concepto de institucionalización. Hablar de lo institucional sin presuponer lo ideológico sería contradictorio, ya que lo primero es una forma de crear visiones (ideológicas) del mundo. Esta afirmación daría mucho que hablar, si bien aquí nos limitamos a constatar que esto se aproxima a una versión institucional marxista de la literatura. El esquema searliano está capacitado por tanto para incluir elementos provenientes de paradigmas teórico-filosóficos muy dispares. En última instancia, Eagleton está poniendo en primer plano el background y network searleanos —él mismo habla de «red»-, subrayando su papel en la identificación de lo literario y mostrando la ideología de la que se nutre este sistema de expectativas y percepción automatizada. De todo esto se derivan todos los rasgos que, como ya hemos visto, caracterizan la visión institucional: el contexto y la actitud de los agentes como único separador entre lo literario y lo no literario, la inexistencia del lenguaje literario y del uso literario del lenguaje o la idea, tomada de Charles Altieri, de que «deberíamos negar el nombre de literatura a una obra que demostrara no ser receptiva a los procedimientos canónicos» (Eagleton, 2013: 84), entre otros muchos ejemplos que cabría presentar.

Podríamos seguir desgranando los mecanismos de la institucionalidad, si bien hemos llegado al objetivo propuesto al mostrar cuáles son los formantes básicos de toda teoría institucional de la literatura y cómo numerosas propuestas pueden entenderse, presentadas bajo el marco conceptual aquí desarrollado, como distintas versiones de esta opción teórica.

\title{
CONCLUSIONES
}

La última teoría searliana es una herramienta de filo cortante en su traslado al campo de la teoría literaria. Hemos intentado mostrar algunos elementos relevantes de cara a esta aplicación, recurriendo en ocasiones al desarrollo que han tenido en la filosofía de la música. Por ello, se ha intentado ubicar la propuesta en el marco de la filosofía del arte y de la música como paso previo necesario para su desarrollo literario. Esto nos ha permitido, entre otras cosas, incorporar las tesis de Dickie, basadas en la distinción entre teorías 
naturales y teorías culturales, y buscar la posibilidad de una definición transcultural, tanto desde un punto de vista social como institucional. En terreno literario, el marco searleano podría esclarecer el estatuto ontológico de las obras literarias, definiéndolas como ontológicamente subjetivas pero epistémicamente objetivas, proporcionando además una terminología estable y claramente delimitada, que goza de reconocimiento en el ámbito filosófico.

Por otro lado, el concepto de intencionalidad nos muestra que toda definición de literatura debe tener en consideración elementos externos al texto. Esto es así incluso en una visión de la literatura como hecho social, lo que nos permitiría salvaguardar el concepto de literariedad y compatibilizarlo con el imprescindible elemento intencional - ergo extrínseco, dependiente de los agentes y sus estados mentales-. Desarrollando y matizando estos moldes, hemos propuesto una visión social de literatura que permite también incluir los llamados casos extremos. Para este análisis, la cuestión de la defectividad se ha revelado a su vez de gran interés y utilidad. Hemos visto asimismo que, tanto desde lo social como desde lo institucional, no es posible una definición de la literatura en términos de condiciones necesarias y suficientes.

Es este último supuesto el que nos ha llevado a adaptar al fenómeno literario el concepto de función - funciones de estatus, funciones agentivas causales- Los términos básicos $(\mathrm{X}, \mathrm{Y}, \mathrm{C})$ de la fórmula constitutiva de todo hecho institucional pueden jugar un distinto rol según las distintas perspectivas institucionales que adoptemos. Una que parece ser común a todas ellas es la consideración de la literatura como hecho institucional iterado que hemos tratado de defender. A su vez, hemos propuesto el concepto de apertura bruta de un hecho institucional como medio de entender el papel secundario que podría tener la literariedad en el marco institucional.

En consecuencia, hemos concluido la necesidad de distinguir entre definiciones institucionalizadas y definiciones institucionales propias, a menudo confundidas, así como la posibilidad de plantear una aproximación institucional transcultural, centrada en la Y de la fórmula constitutiva. Todo este andamiaje conceptual nos permite reubicar y reinterpretar la mayor parte de las propuestas teóricas modernas, desde el formalismo ruso hasta la última producción de un autor como Terry Eagleton.

Dada la imposibilidad de profundizar hasta las últimas consecuencias en todo el espectro temático que posibilita el marco searleano, hemos intentado dejar claro que la propuesta desarrollada en este trabajo encuentra numerosas vías por las que expandirse. Creemos que sería de gran potencial heurístico en otras cuestiones características de la teoría literaria como el problema de los géneros literarios o la cuestión del canon. A pesar de todos los problemas a los que una definición ha de enfrentarse, una cosa parece clara: tiene pleno sentido preguntarse por la ontología de la literatura, y esta indagación puede aportarnos un rédito - una nueva comprensión — tangible. Porque, en definitiva, lejos de acomodarse en ningún dogma o escuela prefijada, la ontología 
social de John Searle nos permite reconsiderar teorías de muy diversa índole y nos proporciona una atalaya firme desde la que contemplar la existencia del fenómeno literario ${ }^{1}$.

\section{BIBLIOGRAFÍA CITADA}

Bobes Naves, M. ${ }^{a}$ del Carmen (2008). Crítica del conocimiento literario. Madrid: Arco Libros.

Davies, Stephen (1991). Definitions of Art, Ithaca. New York: Cornell University Press.

Dickie, George (1997). «Art: Function or Procedure - Nature or Culture?», The Journal of Aesthetics and Art Criticism. 55/1, pp. 19-28.

Dickie, George (2000). «The Institutional Theory of Art», en Noël Carroll (ed.), Theories of Art Today. Wisconsin: The University of Wisconsin Press, pp. 93-108.

Eagleton, Terry (1988). Una introducción a la teoría literaria. México D.F.: Fondo de Cultura Económica. Primera edición de 1983.

Eagleton, Terry (2013). El acontecimiento de la literatura. Barcelona: Ediciones Península.

Eco, Umberto (1971). La definición del arte. Barcelona: Ediciones Martínez Roca. Primera edición de 1968.

Eco, Umberto (2002). Interpretación y sobreinterpretación. Cambridge: Cambridge University Press. Primera edición de 1992.

Fotion, Nick (2000). John Searle. New Jersey: Princeton University Press.

García Leal, José (2002). Filosofía del arte. Madrid: Editorial Síntesis.

Garrido Gallardo, Miguel Ángel (2004). Nueva Introducción a la teoría de la literatura. Madrid: Editorial Síntesis.

Garrido Gallardo, Miguel Ángel, Tzvetan Todorov et al. (1987). La crisis de la literariedad. Madrid: Taurus.

Gracyk, Theodore y Andrew Kania (ed.) (2011). The Routledge Companion to Philosophy and Music. London - New York: Routledge.

Guerreiro, Vitor (2012). «Thinking Clearly About Music», Teorema. XXXI/3, pp. 25-47.

Guijarro Lasheras, Rodrigo (2012). «En busca del concepto musical: tres criterios clave y una cuestión de misterio», Teorema. XXXI/3, pp. 237-252.

Kania, Andrew (2010). «Silent music», The Journal of Aesthetics and Art Criticism. 68, pp.343-353.

Kania, Andrew (2011). «Definition», en Theodore Gracyk y Andrew Kania (ed.), The Routledge Companion to Philosophy and Music. London - New York: Routledge, pp. 3-13.

Lázaro Carreter, Fernando (1999). «La literatura como fenómeno comunicativo», en José Antonio Mayoral (ed.), Pragmática de la comunicación literaria. Madrid: Arco Libros, pp. 151-170. Primera edición del texto de 1980.

Levinson, Jerrold (2011). Music, Art \& Metaphysics. Essays in Philosophical Aesthetics. Oxford: Oxford University Press.

Llovet, Jordi et al. (2005). Teoría literaria y literatura comparada. Barcelona: Editorial Ariel.

Marrades Millet, Julián (2012). «Una perspectiva sobre la filosofía de la música», Teorema. XXXI/3, pp. 5-24.

Ohmann, Richard (1999). «Los actos de habla y la definición de literatura», en José Antonio Mayoral (ed.), Pragmática de la comunicación literaria. Madrid: Arco Libros, pp. 1134. Primera edición del texto de 1971.

\footnotetext{
${ }^{1}$ Deseo agradecerle a Fernando Ángel Moreno sus inteligentes observaciones y comentarios, que hicieron mejorar este trabajo.
} 
Rust, Joshua (2006). John Searle and The Construction of Social Reality. London \& New York: Continuum.

Rust, Joshua (2009). John Searle. London \& New York: Continuum.

Searle, John R. (1992). Intencionalidad: un ensayo en la filosofía de la mente. Madrid: Editorial Taurus.

Searle, John R. (1997). La construcción de la realidad social. Barcelona: Paidós. Primera edición de 1995.

Searle, John R. (2011). Making the Social World. The Structure of Human Civilization. Oxford: Oxford University Press.

Trías, Eugenio (1998). Vértigo y pasión. Madrid: Taurus.

Fecha de recepción: 4 de noviembre de 2013.

Fecha de aceptación: 21 de mayo de 2014. 